\title{
2-convexity and 2-concavity in Schatten ideals
}

\author{
BY G. J. O. JAMESON \\ Department of Mathematics and Statistics, Lancaster University, Lancaster LA1 $4 Y F$
}

(Received 7 August 1995)

\section{Introduction}

The properties $p$-convexity and $q$-concavity are fundamental in the study of Banach sequence spaces (see [L-TzII]), and in recent years have been shown to be of great significance in the theory of the corresponding Schatten ideals ([G-TJ], [LPP] and many other papers). In particular, the notions 2-convex and 2-concave are meaningful in Schatten ideals. It seems to have been noted only recently [LP-P] that a Schatten ideal has either of these properties if the underlying sequence space has. One way of establishing this is to use the fact that if $\left(E,\|\|_{E}\right)$ is 2-convex, then there is another Banach sequence space $\left(F,\|\|_{F}\right)$ such. that $\|x\|_{E}^{2}=\left\|x^{2}\right\|_{F}$ for all $x \in E$. The 2 -concave case can then be deduced using duality, though this raises some difficulties, for example when $E$ is inseparable.

In this note, we present an alternative approach which proceeds directly from the Markus-Mityagin lemma in the spirit of [GK] and [Si], by way of a quadratic variant of the well-known Ky Fan Lemma. As well as being (arguably) a natural route to the result just stated, this approach also delivers a theorem characterizing the norm of an operator $A$ as the supremum (in the 2-convex case) or the infimum (in the 2-concave case) of the norms (in $E$ ) of the sequences $\left(\left\|A e_{j}\right\|\right)$ for orthonormal bases $\left(e_{j}\right)$.

\section{Notation and definitions}

We denote by $x(j)$ the $j$ th term of a numerical sequence $x$, and by $e_{j}$ the $j$ th unit vector. For sequences $x, y$, the product $x y$ and the modulus $|x|$ are defined pointwise in the obvious way. We write $P_{n}$ for the operator (on any sequence space) that replaces all terms after the first $n$ by 0 , so that $P_{n}(x)=\sum_{j=1}^{n} x(j) e_{j}$. By a symmetric Banach sequence space we mean a Banach lattice $\left(E,\|\|_{E}\right)$ of real null sequences with a symmetric norm satisfying further:

(i) $e_{j} \in E$ and $\left\|e_{j}\right\|_{E}=1$ for all $j$,

(ii) $\|x\|_{E}=\lim _{n \rightarrow \infty}\left\|P_{n}(x)\right\|_{E}$ for all $x \in E$.

(We do not exclude the case where $E$ is finite-dimensional.)

Let $(H,\|\|)$ be a separable Hilbert space (of finite or infinite dimension). For a compact operator $A$ on $H$, let $s_{j}(A)(j=1,2, \ldots)$ be the singular numbers of $A$. We denote by $S_{E}(H)$ the Schatten ideal corresponding to the Banach sequence space $E$, with norm $\sigma_{E}$ defined by $\sigma_{E}(A)=\left\|\left(s_{j}(A)\right)\right\|_{E}$.

Let $A_{1}, \ldots, A_{n}$ be self-adjoint elements of $S_{E}(H)$, and let $A_{0}=\left(\sum_{j=1}^{n} A_{j}^{2}\right)^{1 / 2}$. Then $A_{0}$ $\in S_{E}(H)$ (this is most easily seen by considering the operator on $H^{n}$ with first column 
$A_{1}, \ldots, A_{n}$, cf. [LP-P]). Hence one can define (as for sequences spaces) $S_{E}(H)$ to be 2convex if for all such $A_{1}, \ldots, A_{n}$, we have for some $M$

and 2-concave if we have

$$
\sigma_{E}\left(A_{0}\right) \leqslant M\left(\sum_{j=1}^{n} \sigma_{E}\left(A_{j}\right)^{2}\right)^{1 / 2}
$$

$$
\left(\sum_{j=1}^{n} \sigma_{E}\left(A_{j}\right)^{2}\right)^{1 / 2} \leqslant M \sigma_{E}\left(A_{0}\right) .
$$

The least such constant $M$ is, respectively, the 2-convexity or 2-concavity constant of $S_{E}(H)$. We say that $E$, or $S_{E}(H)$, is strictly 2-convex or 2-concave if the constant is 1 . Note that in the above definition it is clearly sufficient to consider positive operators $A_{j}$.

\section{The results}

We will use the two following well-known theorems.

Proposition 1. Let $A$ be compact, and let $\left(e_{i}\right),\left(f_{i}\right)$ be any two orthonormal sets. Then for each $n$,

(i) $\sum_{j=1}^{n}\left|\left\langle A e_{j}, f_{j}\right\rangle\right| \leqslant \sum_{j=1}^{n} s_{j}(A)$,

(ii) $\sum_{j=1}^{n}\left\|A e_{j}\right\|^{2} \leqslant \sum_{j=1}^{n} s_{j}(A)^{2}$.

Statement (i) is essentially [GK, II·4·1]; it also follows in elegant style from [ $\mathbf{S i}$, propositions $1 \cdot 11$ and 1.12], although it is not stated explicitly there. Statement (ii) follows by applying (i) to $A^{*} A$.

For the next result, we denote by $D_{n}$ the dyadic group $\{-1,1\}^{n}$. Elements of $D_{n}$ belong to $\mathbb{R}^{n}$, so act on $\mathbb{R}^{n}$ by multiplication. Also, if $\pi \in S_{n}$, the group of permutations of $\{1,2, \ldots, n\}$ and $x \in \mathbb{R}^{n}$, then $x_{n}$ is the element of $\mathbb{R}^{n}$ defined by $x_{\pi}(j)=x[\pi(j)]$.

Propositron 2. Let $x, y$ be decreasing, non-negative members of $\mathbb{R}^{n}$. Define $X(k)=$ $\sum_{j=1}^{k} x(j)$, and $Y(k)$ similarly. Suppose that $X(k) \leqslant Y(k)$ for each $k$. Then

$$
y \in \operatorname{conv}\left\{\epsilon x_{n}: \epsilon \in D_{n}, \pi \in S_{n}\right\} .
$$

If, further, $X(n)=Y(n)$, then

$$
y \in \operatorname{conv}\left\{x_{\pi}: \pi \in S_{n}\right\} .
$$

Proof. The first statement is the standard Markus-Mityagin lemma (see, for example, [GK, III.3]). The second statement is surely well known: it is stated without proof in [Sch, lemma 4.2], where it is observed that something like it already appears in [HLP]. For completeness, we mention how the proof of $[$ GK] can be adapted for this case. Suppose the statement is false. Then there is a linear functional $\phi$ such that $\phi(y)>\phi\left(x_{\pi}\right)$ for all $\pi \in S_{n}$. Let $\phi(u)=\sum_{j=1}^{n} a_{j} u(j)$. Since $X(n)=Y(n)$, we can add a constant $c$ to each $a_{j}$, and hence we may assume that $a_{j} \geqslant 0$ for each $j$. The proof now proceeds as before, but without the need for terms $\epsilon_{j} \in\{-1,1\}$ to convert negative $a_{j}$ 's to $\left|a_{j}\right|$. 
We deduce a quadratic variant of the Ky Fan lemma.

Proposition 3. Let $E$ be a Banach sequence space. Let $x, y$ be decreasing, non-negative null sequences such that $\sum_{j=1}^{k} y(j)^{2} \leqslant \sum_{j=1}^{k} x(j)^{2}$ for all $k$. Then:

(i) if $E$ is strictly 2-convex and $x \in E$, then $y \in E$ and $\|y\|_{E} \leqslant\|x\|_{E}$;

(ii) if $E$ is strictly 2-concave, $y \in E$ and also $\sum_{j=1}^{o} y(j)^{2}=\sum_{j=1}^{\infty} x(j)^{2}$, then $x \in E$ and $\|y\|_{E} \geqslant\|x\|_{E}$.

Proof. It is clearly enough to prove both statements for finitely non-zero sequences $x, y$ : the statement is then obtained by considering limits (with a small adjustment to the $n$th term to ensure the required equality in case (ii)).

(i) By the first statement in Proposition 2, there exist rearrangements $z_{r}$ of $x$ (for $1 \leqslant r \leqslant R$, say), $\lambda_{r}>0$ and $\epsilon_{r} \in D_{n}$ such that $\sum_{r=1}^{R} \lambda_{r}=1$ and $y^{2}=\sum_{r=1}^{R} \lambda_{r} \epsilon_{r} z_{r}^{2}$, hence $y^{2} \leqslant \sum_{r=1}^{R} \lambda_{r} z_{r}^{2}$. By 2-convexity and the fact that $\left\|z_{r}\right\|_{E}=\|x\|_{E}$ for each $r$, we have

$$
\begin{aligned}
\|y\|_{E}^{2} & \leqslant \sum_{r=1}^{R} \lambda_{r}\left\|z_{r}\right\|_{E}^{2} \\
& =\|x\|_{E}^{2} .
\end{aligned}
$$

(ii) By the second statement in Proposition 2, there exist $z_{r}$ and $\lambda_{r}>0$ such that $\sum_{r=1}^{R} \lambda_{r}=1$ and $y^{2}=\sum_{r=1}^{R} \lambda_{r} z_{r}^{2}$. 2-concavity gives the stated inequality.

Lemma 1. If the Banach sequence space $E$ is strictly 2-concave, then $E$ is contained in $l_{2}$ and $\|x\|_{2} \leqslant\|x\|_{E}$ for all $x \in E$.

Proof. Take $x \in E$. Since $\left(E,\|\|_{E}\right)$ is a Banach lattice, $\left\|P_{n} x\right\|_{E} \leqslant\|x\|_{E}$ for each $n$. Write $x_{j}=x(j) e_{j}$. Then $\left(P_{n} x\right)^{2}=\sum_{j=1}^{n} x(j)^{2} e_{j}=\sum_{j=1}^{n} x_{j}^{2}$, so by 2-concavity,

$$
\sum_{j=1}^{n} x(j)^{2}=\sum_{j=1}^{n}\left\|x_{j}\right\|_{E}^{2} \leqslant\left\|P_{n} x\right\|_{E}^{2} \leqslant\|x\|_{E}^{2} .
$$

The statement follows.

In the same way, if $E$ is 2-convex, then $E$ contains $l_{2}$ and $\|x\|_{E} \leqslant\|x\|_{2}$.

It is now easy to characterize the Schatten ideal norm of an operator in the way stated in the introduction. The following result is well known for the classical ideals $S_{p}(H)$ given by $E=l_{p}$ (see, for example, [GK], p. 95).

Theorem 1. Let $A$ be an element of $S_{E}(H)$.

(i) If $E$ is strictly 2-convex, then for any orthonormal set $\left(e_{j}\right)$,

$$
\left\|\left(\left\|A e_{j}\right\|\right)\right\|_{E} \leqslant \sigma_{E}(T) .
$$

(ii) If $E$ is strictly 2-concave, then for any orthonormal basis $\left(e_{j}\right)$,

$$
\sigma_{E}(A) \leqslant\left\|\left(\left\|A e_{j}\right\|\right)\right\|_{E}
$$

whenever the right-hand side is finite.

In both cases, equality occurs for the $\left(e_{j}\right)$ appearing in the spectral representation of $A$.

Proof. We may assume that $\left(e_{j}\right)$ is ordered so that $\left(\left\|A e_{j}\right\|\right)$ is decreasing. Statement (i) follows at once from Proposition 1 (ii) and Proposition 3 (i). If $E$ is 2 -concave, then 
by Lemma $1, E \subseteq l_{2}$, so $A \in S_{2}(H)$ (the Hilbert-Schmidt operators) and for any orthonormal basis $\left(e_{j}\right)$,

$$
\sum_{j=1}^{\infty}\left\|A e_{j}\right\|^{2}=\sum_{j=1}^{\infty} s_{j}(A)^{2}
$$

Proposition 3(ii) now gives statement (ii).

We remark that elementary examples (e.g. with $E=l_{1}$ ) show that the right-hand side in statement (ii) is not always finite.

A further application of 2-convexity or 2-concavity now yields the result stated at the beginning.

Theorem 2. If $E$ is strictly 2-convex or 2-concave, then so is $S_{E}(H)$.

Proof. Let $A_{1}, \ldots, A_{n}$ be positive elements of $S_{E}(H)$, and let $A=\left(\sum_{i=1}^{n} A_{i}^{2}\right)^{1 / 2}$. Let the spectral representation of $A$ be $\sum_{j=1}^{\infty} \mu_{j} e_{j} \otimes e_{j}$, so that $\mu_{j}=s_{j}(A)$ and $A e_{j}=\mu_{j} e_{j}$, hence

$$
\mu_{j}^{2}=\left\langle A^{2} e_{j}, e_{j}\right\rangle=\sum_{i=1}^{n}\left\langle A_{i}^{2} e_{j}, e_{j}\right\rangle=\sum_{i=1}^{n}\left\|A_{i} e_{j}\right\|^{2} .
$$

Define scalar sequences $a, a_{i}$ by:

$$
\begin{aligned}
a(j) & =\mu_{j}, \\
a_{i}(j) & =\left\|A_{i} e_{j}\right\| .
\end{aligned}
$$

Then $a^{2}=\sum_{i=1}^{n} a_{i}^{2}$ and $\|a\|_{E}=\sigma_{E}(A)$.

If $E$ is 2-convex, then $\|a\|_{E}^{2} \leqslant \sum_{i=1}^{n}\left\|a_{i}\right\|_{E}^{2}$ and Theorem 1 (i) gives $\left\|a_{i}\right\|_{E} \leqslant \sigma_{E}\left(A_{i}\right)$, hence

$$
\sigma_{E}(A)^{2} \leqslant \sum_{i=1}^{n} \sigma_{E}\left(A_{i}\right)^{2}
$$

as required. If $E$ is 2-concave, the same applies with both inequalities reversed.

2-convexity and 2-concavity constants. There are plenty of examples of Banach sequence spaces that are 2-convex or 2-concave, but not with constant 1, for example: (i) finite-dimensional spaces in general, (ii) certain Lorentz sequence spaces (see $[\mathbf{R}]$, [J]). If $E$ has 2 -convexity or 2-concavity constant $M(\neq 1)$, then clearly Proposition 3 and Theorem 1 hold with the constant $M$ inserted. Owing to the second use of 2-convexity or 2-concavity in Theorem 2, the above method requires the insertion of $M^{2}$ in this Theorem. Actually, $S_{E}(H)$ has the same 2-convexity or 2concavity constant as $E$. To show this, we amend the method as follows. With $a(j)=s_{j}(A)$ as above, Proposition 1 gives

$$
\sum_{j=1}^{k} a(j)^{2}=\sum_{j=1}^{k} \sum_{i=1}^{n}\left\|A_{i} e_{j}\right\|^{2} \leqslant \sum_{i=1}^{n} \sum_{j=1}^{k} s_{j}\left(A_{i}\right)^{2} .
$$

Write $b_{i}(j)=s_{j}\left(A_{i}\right)$. The stated result is now given by the following variant of Proposition 3:

Proposition 4. Let $E$ be a Banach sequence space. Let $a, b_{1}, \ldots, b_{n}$ be decreasing non-negative sequences belonging to $E$, and let $b^{2}=\sum_{i=1}^{n} b_{i}^{2}$. Suppose that $\sum_{j=1}^{k} a(j)^{2} \leqslant$ $\sum_{j=1}^{k} b(j)^{2}$ for all $k$. Then:

(i) if $E$ has 2-convexity constant $M$, then $\|a\|_{E}^{2} \leqslant M^{2} \sum_{i=1}^{n}\left\|b_{i}\right\|_{E}^{2}$; 
(ii) if $E$ has 2-concavity constant $M$ and also $\sum_{j=1}^{\infty} a(j)^{2}=\sum_{j=1}^{\infty} b(j)^{2}$, then $\sum_{i=1}^{n}\left\|b_{i}\right\|_{E}^{2} \leqslant M^{2}\|a\|_{E}^{2}$.

Proof. Again it is enough to consider the finite-dimensional case. As in Proposition 3 , there exist $\lambda_{r}>0$ (for $r=1, \ldots, R$ ) and $\pi_{r} \in S_{n}$ such that $\sum_{r=1}^{R} \lambda_{r}=1$ and

$$
a^{2} \leqslant \sum_{r=1}^{R} \lambda_{r} b_{\pi_{r}}^{2}=\sum_{r=1}^{R} \sum_{i=1}^{n} \lambda_{r} b_{i, \pi_{r}}^{2}
$$

with the $\leqslant$ replaced by equality in case (ii). Both statements now follow.

\section{REFERENCES}

[G-TJ] D. J. H. GaRLING and N. TomczaK-JAEgermanN. The cotype and uniform convexity of unitary ideals. Israel J. Math. 45 (1983), 175-197.

[GK] I. C. GokHBERG and M. G. KREIN. Introduction to the theory of linear nonselfadjoint operators (Amer. Math. Soc., 1969).

[HLP] G. H. Hardy, J. E. Littlewood and G. Pólya Inequalities (Cambridge Univ. Press, 1934).

[J] G. J. O. JAMESON. The $q$-concavity constants of Lorentz sequence spaces, preprint.

[L-Tz II] J. Lindenstrauss and L. Tzafriri. Classical Banach spaces II (Springer, 1979).

[LP-P] F. Lust-Piquard and G. Pisier. Non-commutative Khintchine and Paley inequalities. Arkiv för Matematik 29 (1991), 241-260.

[R] S. REISNER. A factorization theorem in Banach lattices and its application to Lorentz spaces. Ann. Inst. Fourier 31 (1981), 239-255.

[Sch] C. Schürt. Lorentz spaces that are isomorphic to subspaces of $L_{1}$. Trans. Amer. Math. Soc. 314 (1989). 583-595.

[Si] B. Simon. Trace ideals and their applications (London Math. Soc., 1979). 
